\title{
Oxygen-ozone therapy in arthroplasty infections
}

\author{
Alessandra Vittoria De Lisi, Marianno Franzini, Giovanni Ricevuti \\ Italian Society for Oxygen-Ozone Therapy (SIOOT), Gorle (BG), Italy
}

\begin{abstract}
The prevention of surgical site infections is a priority within the objectives of the National Health System. The negative consequences of postoperative infections (morbidity, duration of hospitalization, mortality) are particularly serious events for the individual patient and for the community, given both the welfare resources needed to treat them, and the continued growth of the phenomenon of antibiotic resistance.

In a global health context in which evidence-based medicine has established itself, all medical interventions must be reconsidered in the light of what can be inferred from the literature evaluated according to strict methodological criteria, all the more so when the evidence is available and unanimously shared.

The future objective will be to introduce in the national guidelines a protocol involving the use of ozone therapy alongside antibiotic therapy, both in the prevention and treatment of infections, to avoid the emergence of antibiotic resistance and thus reduce the expenditure of the national health system.
\end{abstract}

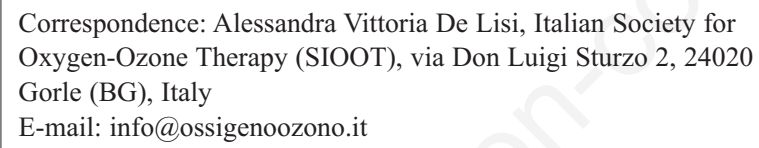

E-mail: info@ossigenoozono.it

Key words: Arthroplasty infections; Surgical site infections; Oxygenozone therapy.

Contributions: the authors contributed equally.

Conflict of interest: the authors declare no potential conflict of interest.

Funding: none.

Received for publication: 1 March 2019.

Accepted for publication: 25 March 2019.

CCopyright A.V. De Lisi et al., 2019

Licensee PAGEPress, Italy

Ozone Therapy 2019; 4:8187

doi:10.4081/ozone.2019.8187

This article is distributed under the terms of the Creative Commons Attribution Noncommercial License (by-nc 4.0) which permits any noncommercial use, distribution, and reproduction in any medium, provided the original author(s) and source are credited.

\section{Introduction}

In the specific area of prevention of infections in orthopaedic surgery, the search for evidence to support the procedures undertaken to prevent them fortunately clashes with the current relatively low incidence of infection, i.e. $0.5-2 \%$ in elective surgery (Figure 1).

Most infections occur during or immediately after surgery as a result of failure of the surgical wound or infection; less frequently, joint infections develop late after implantation of the prosthesis by direct inoculation or hematogenic diffusion. The onset can be acute with fever, pain and local signs of inflammation, especially in case of infection with Staphylococcus aureus, pyogenic streptococci, and enterobacteria. Alternatively, when less virulent bacteria such as diphtheroids or coagulase-negative streptococci are involved, the infection may persist for months or years without causing appreciable symptoms. These paucisymptomatic infections are generally contracted during the implantation of the prosthesis and are discovered during the evaluation of unexplained chronic pain and following the radiological finding of the detachment of the prosthesis; in these cases, the Erythrocyte Sedimentation Rate (ESR) and the C-Reactive Protein (CRP) are usually high (Figure 2).

In addition, the arthrocentesis of the joint concerned allows an accurate diagnosis: the cell count shows a pleiocytosis of the synovial fluid with a prevalence of polymorphonuclear leukocytes, highly suggestive of infectious arthritis, followed by the culture test, for aerobes, anaerobes and fungi.

To prevent the disastrous consequences of an infection, patients who are candidates for the implantation of a joint prosthesis must be carefully selected and prepared. The frequency of infections, in fact, is particularly high in patients with rheumatoid arthritis, diabetes mellitus, in subjects previously undergoing surgery on the joint and in individuals suffering from diseases that require the use of immunosuppressants. ${ }^{1,2}$

Patients are advised to preoperatively wash antiseptically the day or evening before surgery to reduce the bacterial load on the skin, using antimicrobial soap or antiseptic solution. Where trichotomy is required, it is recommended to limit it to the incision area, use an electric razor (avoid blade razors) and perform it on the day of surgery, if possible before entering the operating room.

It is recommended that blood sugar levels be maintained at $<200 \mathrm{mg} / \mathrm{dL}$ during the perioperative period to reduce the risk of surgical site infections in all patients, diabetics and non-diabetics.

Antibiotic prophylaxis is recommended for orthopedic and traumatological surgery requiring the implantation of devices (prostheses, means of synthesis, biomaterials) in the open air, while in other cases it is to be considered from time to time according to the invasiveness of the procedure and the individual characteristics of the patient.

The choice of antibiotic involves cephalosporins of I-II gener- 
ation, alternatively glycopeptides or clindamycin in case of allergy or high incidences/high risk of Methicillin-resistant $S$. aureus infection. In addition, the association with antibiotics that are effective against negative Grams in particular local contexts/situations must be considered. In addition, the guidelines recommend the use of alcoholic antiseptic solutions for the preparation of the surgical site in the operating room when preparing the field.

All these measures have made it possible to lower the frequency of postoperative infections in many centers to less than $1 \%$.

Much work remains to be done, to ensure the least possible risk for our patients, and we must start immediately, to reduce that percentage, albeit minimal, but which has serious and devastating consequences for the patient and, a very topical issue, is going to aggravate the national health system.

And this is where oxygen-ozone therapy comes into play, which is a therapy based on the delivery of a certain amount of ozone in the body, through various techniques that on some diseases, has allowed to obtain important therapeutic results, thanks to: i) reactivation of the microcirculation, increasing the availability of oxygen to the tissues and reducing the blood viscosity; ii) antioxidant action; iii) anti-inflammatory, analgesic and muscle relaxant action; iv) antibacterial effect, fungicide and static virus.

Thanks to the latter effect, it was introduced in the infectious field, especially in the field of antibiotic resistance. ${ }^{3}$

Antibiotic resistance, which is the ability of a micro-organism

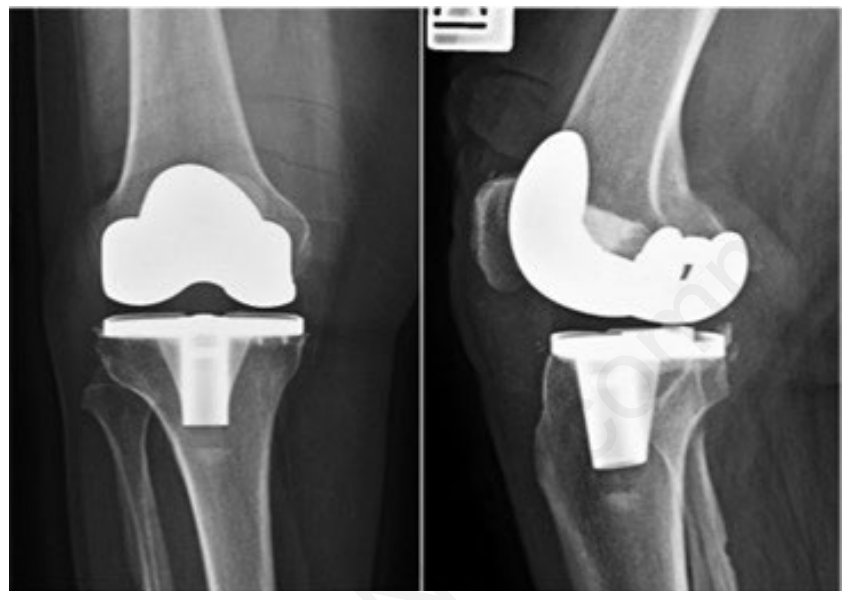

Figure 1. Radiographic image of a knee prosthesis.

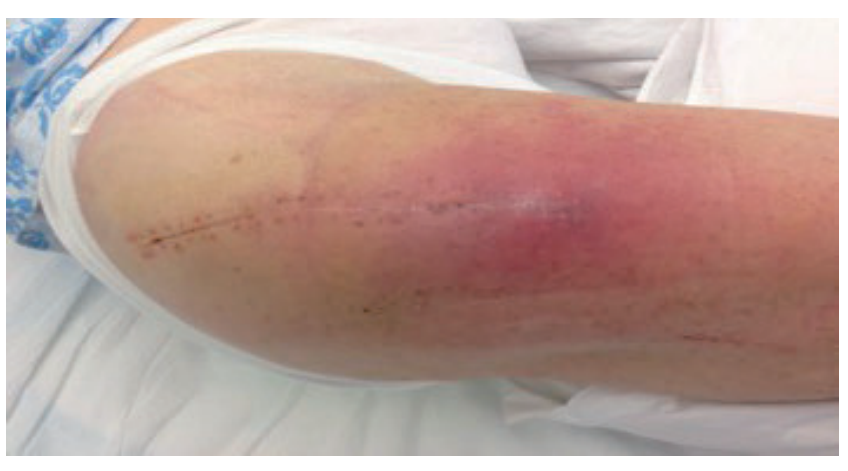

Figure 2. Clinical signs of prosthetic infection. to resist the action of one or more antimicrobial agents, is one of the greatest limitations of effective antibacterial chemotherapy. ${ }^{2,4}$

The effectiveness of antibiotics is undermined by a growing number of antibiotic-resistant pathogens and is implicated in a high frequency of mortality and morbidity, as well as in increasing treatment costs, therefore considered one of the major threats to global public health. ${ }^{5}$

If a widespread epidemic were to occur, we can expect around 200,000 people with a bacterial blood infection that cannot be treated effectively with the drugs available to us today. ${ }^{6}$ Antibiotic resistance may develop as a result of selective pressure exerted by antibiotics, may be induced by expansion of strains with resistance mutations or may be the result of acquisition of resistance genes by means of a horizontal gene transfer mechanism. ${ }^{7}$ Ozone is a powerful oxidant of microorganisms and an important disinfectant. ${ }^{8}$ The kinetics of disinfection is described by Chick-Watson's law: $\mathrm{k}=$ C.t. Where $\mathrm{k}=$ constant reaction, depends on the type of microorganism and the disinfectant, $\mathrm{c}=$ concentration of the disinfectant in $\mathrm{mg} / \mathrm{l}, \mathrm{t}=$ contact time (minutes) between the disinfectant and water needed to deactivate a microorganism.

Ozone is an allotropic form of oxygen, composed of three atoms of oxygen, normally produced by the immune system all living beings. It was discovered in 1840 by Van Maum and Schobein and used by Von Simens to disinfect water. However, it was in 1902 that J.H. Clark, in the Dictionary of Medical Practice, described its favorable effects on infections, diabetes and cancer and in the 1930s Payr extended its use in surgical pathologies. ${ }^{9}$ It has been reported that ozone can be used as a bactericidal agent in various forms, e.g. ozonized salt solution, ozonized water, ozonized oil and more frequently $\mathrm{O}_{2} / \mathrm{O}_{3}$ mixture. ${ }^{8}$ Many studies on oxygen-ozone therapy have established that ozone, when used in vitro, can destroy all types of bacteria, both gram positive and gram negative. In addition, it has been suggested that the sensitivity of microorganisms to antibiotics increases when ozone is also used, and also increases the sensitivity of microorganisms to the action of the immune complement system. ${ }^{10}$ In summary, the reason for the ozone's ability to inactivate viruses and bacteria lies in its high oxidative potential, which in turn originates directly from the structure of the ozone molecule. ${ }^{11}$ Cell membranes are the first target of ozone. Ozone-induced changes in the intracellular content (oxidation of plasma proteins, alterations in cellular functions) are probably caused by the action of secondary oxidizing agents, produced by the lysis of membrane lipids. In fact, through the production of Reactive Oxygen Species (ROS) $\left(\mathrm{H}_{2} \mathrm{O}_{2}\right.$, $\mathrm{OH}, \mathrm{O}_{-2}$ ), ozone acts as an oxidant in a similar way to what happens in leukocytes activated during phagocytosis. It is plausible to imagine that this increase is responsible for the bactericidal effect of ozone both aerobic and anaerobic bacteria, considering that the latter are without enzymes, such as superoxide dismutase, able to neutralize the oxidizing action of these free radicals. In fact, studies ${ }^{12}$ have shown that the mechanism underlying this bactericidal effect occurs indirectly through the interaction of ROS with the enzymatic structures of leukocytes. In particular, it seems that $\mathrm{H}_{2} \mathrm{O}_{2}$, which occurs after blood ozonisation, is responsible for the activation of the Nuclear Factor-KB, necessary for the activation of gene transcription for different cytokines (INF-beta, TNF alpha, IL-2, IL-6) in T lymphocytes and monocytes. ${ }^{12}$ It has also been shown that antibodies catalyze the generation of hydrogen peroxide from molecular oxygen and water: this process can lead to the efficient killing of bacteria, regardless of the antigenic specificity of the antibody. It has been suggested that the oxidation pathway of water catalyzed by antibodies produces an additional molecular species with a chemical signature similar to that of ozone. This species is generated during oxidative stress of activated human neutrophils and during inflammation. These observations suggest that there are alternative ways to 
kill bacteria that are mediated by powerful oxidizers previously unknown to biology. ${ }^{13}$

Local destruction of the membranes is the direct cause of the elimination of bacteria; the bacterial cell loses its ability to live, reproduce or both. The effect of ozone on the micelles of pathogenic fungi initially modifies the external structure of these (the cytoplasmic membrane) and, subsequently, compromises the intracellular membranes. As a result of this action, the hyphae of the pathogenic fungi flatten and wrinkled, with the appearance of cell wall defects until the complete destruction of all components of the structure of the fungi. The viricidal mechanisms are more complex. The action of ozone molecules leads to several results: partial destruction of the viral envelopes and loss of its properties; inactivation of intracellular reverse transcriptase with the consequent inhibition of the processes of transcription and synthesis of proteins and, therefore, of the formation of new viral particles; alteration of the ability of viruses to combine with the receptors of the target cells. In addition, ozone has been shown to destroy both extracellular and intracellular viruses. ${ }^{10}$

\section{Materials and Methods}

A study carried out in the outpatient oxygen-ozone therapy clinic of Bergamo, Italy was analyzed. Ozone, here, is generated with Medical 95 Computerized Photometric System produced by Multiossigen. With regard to the above, three patients were selected from this study who had undergone prosthetic revision surgery and could no longer undergo new surgery. One patient replaced the shoulder joint with prostheses, one patient underwent two prosthetic revision surgeries on the left knee and the third reached the outpatient clinic after four left knee orthopedic surgeries (Figure 3).

Patients started ozone therapy after failing to heal the infectious disease treated with multiple cycles of broad-spectrum antibiotics. In all cases a therapy with auto-hemoinfusion and concentrations between 30 and 50 micrograms was chosen; the mixture of oxygen-ozone was infused in the outpatient clinic for a number of sessions selected on the basis of the response to the therapy and the extension of the lesion/pathology at the beginning of therapy. The number of auto-hemoinfusion sessions varies from a minimum of 3 sessions to a maximum of 30 (Figure 4).

Patients were also treated with subcutaneous infiltrations of oxygen-ozone mixture in the area of skin lesion, with a concentration of 2-3 micrograms for each microinjection of 5/10 cc. They were injected 100 to $150 \mathrm{cc}$ per limb. Two patients were treated with rectal oxygen-ozone insufflation, with a concentration of 20 to 30 micrograms and an amount of $150 \mathrm{cc}$ per session. Patients with ulcerative skin lesions extended to the lower limbs with pain and considerable loss of substance were also treated with a slightly hyperbaric bag, for a therapy of about 15 minutes per limb. ${ }^{3}$

\section{Results}

The effects of oxygen-ozone therapy by means of large autohemoinfusion (GAE), associated with subcutaneous injections, are represented by a considerable disinfection, if not total disappearance of the germs involved in the infectious disease. The results of this study showed that the application of ozone prevents the worsening of skin lesions and improves the healing of infected tissues. Moreover, when ozone is used with self-infusion, the patient's infectious state improves with an optimal result of the values of CRP and ESR. In addition, it has been evaluated how 2-3 weekly sessions of oxygen-ozone therapy can give better results than a treatment with a single weekly session, because it can maintain high immune factors. Even the use of average concentrations, about 40-50 micrograms, are preferable to higher or lower concentrations. This way you get the best response from the immune system. In fact, it is possible to notice how the CRP values were reduced until the frequency of therapies was two per week; when it was decided to reduce the frequency to a single weekly therapy, the CRP values were again increased. The experience of this study suggests that, in order to bring about a definitive resolution of the disease, two sessions of GAE per week are necessary for at least one month after the consolidation of the objective and subjective result. ${ }^{3}$

In this context, it should be remembered that the bactericidal activity of ozone is demonstrated when, in vitro, there is a dilution of $1 / 1000$ from the bacterium broth culture; if, on the other hand, dilutions of $1 / 100$ or $1 / 10$ are obtained from the broth culture, the activity of ozone has only a bacteriostatic effect with the growth of the bacterium, again, after 24-30 hours from the inoculation of the mixture of oxygen-ozone. ${ }^{12}$ Then we can assume that high bacterial loads need more frequent treatment with oxygen-ozone to achieve the bactericidal effect, with the cancellation of bacterial growth. It should also be noted that a patient was suffering from chronic lymphatic leukemia; the examinations did not show any worsening of the disease. Therapy is therefore not contraindicated in the presence of such pathologies of the hematopoietic system. In general, the hematological picture of no patient has changed as a result of auto-hemoinfusion therapy. ${ }^{3}$

\section{Discussion}

It has been suggested by many studies that ozone can support antibiotic therapy and increase the benefits in the treatment of infectious diseases.

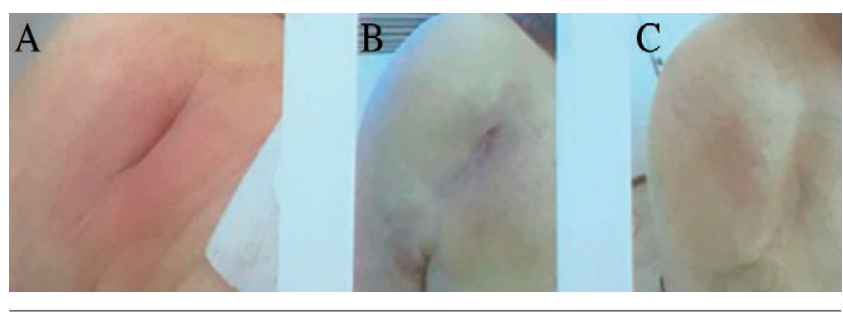

Figure 3. Infection of a shoulder prosthesis. (A) December 23, 2014: CRP=23; (B) 2 weeks later; (C) 18 months later: $C R P=1$.

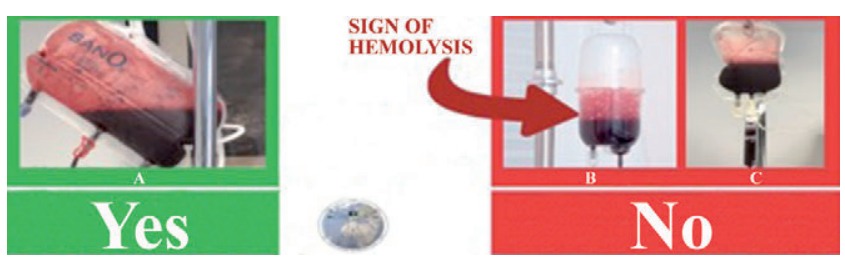

Figure 4. Auto-hemoinfusion with SANO3 bag, certified by the Italian Ministry of Health and SIOOT protocols. (A) Benefits of auto-hemoinfusion with SANO3: it does not release phthalates nor create hyperbarism, which produces hemolysis (rupture of red blood cells); (B) a glass or plastic hyperbaric bowl releases phthalates and creates hemolysis; $(C)$ the bag releases phthalates in blood; it can produce hemolysis. 
For example, Gullmen et al. demonstrated that simultaneous application of vancomycin and ozone was effective in improving mediastinitis related to methicillin-resistant $S$. aureus infection. These results suggest that ozone has an antimicrobial activity and can complement the standard antibiotic regimen by increasing the elimination of bacteria in mediastinitis caused by methicillin-resistant $S$. aureus. ${ }^{14}$ Daulbaeva et al. have shown that oxygen-ozone therapy in skin lesions of the face and neck significantly increased the sensitivity of microorganisms to antibiotics in $79 \%$ of cases. ${ }^{15}$ Belianin et al. demonstrated that adding ozone infusions to antibiotic therapy for 4 months eliminated resistance of isolated strains of Mycobacterium Tubercolosis to isoniazid and/or rinfampicin. ${ }^{16}$ Therefore, in accordance with the data in the literature, in this study it was decided to administer oxygen-ozone to patients with chemotherapy-resistant infections. In all cases the therapy was useful to control the disease - regardless of the microorganism involved - and the resolution was confirmed by the objective examination and laboratory results. ${ }^{3}$

\section{Conclusions}

Repeating treatment for refractory infections requires a prolonged cycle (at least 4 months) with a combination of several antibiotics. $^{2}$ In 2009, the Infectious Disease Society of America took stock of research into new antibiotics and discovered only 16 antibiotics in development. ${ }^{17}$

As the phenomenon of antimicrobial resistance is on the rise, ozone is considered to be a real possibility in the future for the treatment of infections that do not respond adequately to treatment with antibiotics. ${ }^{3}$

Given my daily work in the orthopedic field (in particular knee and hip prosthetics) and considering that in the department where I work flow patients with prosthetic infections from all over Italy, I promise to be part of that group of ozone-therapists that will help to increase the volume of data available so far.

A first step could be to perform a study to evaluate the treatment of two distinct groups of patients undergoing arthroplasty surgery: the first group (control) treated according to the prophylactic guidelines of antibiotic (cefazolin $2 \mathrm{~g}$ in the operating room and cefazolin $2 \mathrm{~g}$ after 6 hours from the first administration, associated with vancomycin if diabetic or immunosuppressed); the second group treated according to the prophylactic guidelines of antibiotic (mentioned above) and by auto-hemoinfusion of oxygen-ozone prophylactic therapy. The aim of the study will therefore be to demonstrate the possible lower incidence of post-operative infections in the group treated with antibiotic prophylaxis and oxygen-ozone prophylactic therapy.

A concomitant step in the research could be to expand the existing literature on the treatment of prosthetic infections by oxygen-ozone therapy associated with the antibiotic of choice, especially in order to achieve a therapeutic plan that can serve as guidelines for the future.

\section{References}

1. Linea Guida SIOT. Prevenzione delle infezioni in chirurgia ortopedica. Gior Ital Ortop Traumatol 2018;44:6-29. Available from: http:/www.giot.it/article/linea-guida-siot-prevenzionedelle-infezioni-in-chirurgia-ortopedica/

2. Jameson JL, Fauci AS, Kasper DL, et al. Harrison's principles of internal medicine. 18th ed. New York: McGraw-Hill; 2012.

3. Franzini M, Valdenassi L, Ionita G. First evaluations of oxygen-ozone therapy in antibiotic-resistant infections. Ozone Ther 2016;1:5-7.

4. Antimicrobial resistance surveillance in Europe. Annual Report of the European Antimicrobial Resistance Surveillance Network (EARS - Net), 2014. European Centre for Disease Prevention and Control, Stockholm 2015. Available from: ecdc.europa.eu/en/publications/Publications/antimicrobialresistance-europe-2015.pdf

5. Lin J, Nishino K, Roberts MC, et al. Mechanisms of antibiotic resistance. Front Microbiol 2015;6:1-3.

6. National Risk Register of Civil Emergencies - 2015 edition. London: Cabinet Office; 2015. Available from: www.officialdocuments.gov.uk

7. Webber MA, Whitehead RN, Mount M, et al. Parallel evolutionary pathways to antibiotic resistance selected by biocide exposure. J Antimicrob Chemother 2015;70:2241-8.

8. Fontes B, Cattani Heimbecker AM, de Souza Brito G, et al. Effect of low-dose gaseous ozone on pathogenic bacteria. BMC Infect Dis 2012;12:358.

9. Simonetti V, Liboni W, Speciani L. Ozonoterapia e nutrizione nell'infiammazione e degenerazione. Torino: Edison Minerva Medica; 2018.

10. Schwartz A, Kontorschikova CN, Malesnikov OV, et al. Guia para el uso medico del ozono. Madrid: Aepromo; 2011.

11. Rilling S, Viebahn R. The use of ozone in medicine. 2nd rev ed., 1st Eng ed. Heidelberg: Haug Publisher; 1987.

12. Luongo C, Lettieri A, Sammartino A, et al. Effetto antimicrobico dell'ozono su colture di Pseudomonas Aeruginosa e Staphylococco Aureus. Riv Ital Ossigeno-Ozonoterapia 2002;1:13-8.

13. Wentworth P, Nieva J, Jones T, et al. Evidence for antibodycatalyzed ozone formation in bacterial killing and inflammation. Science 2002;298:2195-9.

14. Gulmen S, Kurtoglu T, Meteoglu I, et al. Ozone therapy as an adjunct to vancomycin enhances bacterial elimination in methicillin resistant Staphylococcus aureus mediastinitis. J Surg Res 2013;185:64-9.

15. Daulbaeva AA, BaizaKova GT. Effect of ozone on antibiotic sensitivity of microorganisms. Stomatologiia 2003;82:36-8.

16. Belianin II, Shmelev EI. Changes in drug resistance of Mycobacteria in the simultaneous use of chemottherapy and intravenous infusions of dissolved ozone. Probl Tuberk Bolezn Legk 2004;7:32-5.

17. McKenna M. Il nemico dentro. Le Scienze 2011;514:58-65. 